\title{
Coordinate involvement of Nodal-dependent inhibition and Wnt-dependent activation in the maintenance of organizer-specific bmp2b in zebrafish
}

\author{
YU XUE ${ }^{1, \#,}$ CENCAN XING $2,3, \#$, WENJUAN ZHANG ${ }^{1}$, CANBIN CHEN ${ }^{1}$, \\ JINGJIN XU ${ }^{1}$, ANMING MENG ${ }^{*}, 3$ and YUTIAN PAN*,1
}

\begin{abstract}
${ }^{1}$ The Engineering Technological Center of Mushroom Industry, Minnan Normal University, Zhangzhou, Fujian, ${ }^{2}$ Institute of Agro-products Processing Science and Technology, Chinese Academy of Agricultural Sciences, Beijing and ${ }^{3}$ State Key Laboratory of Biomembrane and Membrane Engineering, Tsinghua-Peking Center for Life Sciences, School of Life Sciences, Tsinghua University, Beijing, China
\end{abstract}

\begin{abstract}
A vertebrate signaling center, known in zebrafish as the organizer, is essential for axis patterning and formation and is regulated by multiple cell signaling pathways, including Wnt, Nodal, and Bmp. Organizer-specific Bmp2b plays important roles in the maintenance of the Bmp activity gradient and dorsal-ventral patterning. However, it is unknown how transcription of $b m p 2 b$ in the organizer is regulated. In this study, we generated a bmp2b transgenic line Tsg($2.272 \mathrm{bmp} 2 \mathrm{~b}: \mathrm{gfp}$ /that reproduced organizer-specific bmp2b expression. Dissection analysis revealed that a $0.273-\mathrm{kb}$ minimal promoter was indispensable for $b m p 2 b$ expression in the dorsal organizer. Reporter assays showed that organizer-specific $b m p 2 b$ is negatively regulated by the Nodal signal and positively regulated by the Wnt signal in both embryos and cell lines. Promoter analysis and chromatin-immunoprecipitation (ChIP) indicated that one consensus Smad-binding element (SBE) (CAGAC) and one Lef/Tcf-binding element (LBE) (AGATAA) were present in the $\mathbf{0 . 2 7 3 - k b}$ promoter, and could be directly bound by Smad2 and $\beta$-catenin proteins. Together, these results suggest that maintenance of organizer-specific $b m p 2 b$ expression involves opposite and concerted regulation by Nodal and Wnt signaling.
\end{abstract}

KEY WORDS: zebrafish, organizer-specific bmp2b, transcriptional regulation, Nodal, Wnt

\section{Introduction}

A signaling center known as the organizer in zebrafish plays an essential role in germ layer formation and body axis patterning during early embryogenesis. Embryological manipulations have confirmed that analogous signaling centers are ubiquitously present in vertebrate embryos, and are known as "Spemann Organizer" in Xenopus, "Hensen's node" in chicken, and "node" in mouse. These signaling centers are responsible for controlling cell fate, and studies using transplant experiments have demonstrated that their activity can induce a second body axis (Harland and Gerhart 1997; Kelly et al., 2000; Medina et al., 1997; Schier 2001; Yasuo and Lemaire 2001).

In zebrafish, the organizer forms during the shield stage at 6 hours postfertilization (hpf) in a process that involves regulation by members of multiple cell signaling pathways. Among these, Wnt signal plays an important role during dorsal organizer generation. Recent study reported that, the maternal Wnt8a which functions as the dorsal determinant, is required for the primary dorsal center localization (Lu et al., 2011), while the zygotic Wnt signal restricts

Abbreviations used in this paper: ChIP, chromatin-immunoprecipitation; LBE, Lef/ Tcf-binding element; SBE, smad-binding element.

\footnotetext{
*Address correspondence to: Anming Meng. Room C121, Medicine Building,Tsinghua University, Haidian District, Beijing City, China, 100084. Tel: 86-10-6277-2256. E-mail: mengam@mail.tsinghua.edu.cn or Yutian Pan. Room 603, DaliTeaching Building, Minnan Normal University, Xiangcheng District, Zhangzhou City, Fujian,
} China, 363000. Tel: 86-596-252-3230. E-mail: pytmnnu@163.com

\#Note:These authors contributed equally to this work.

Supplementary Material (one figure) for this paper is available at: http://dx.doi.org/10.1387/ijdb.150193yx

Accepted: 5 November 2015.

ISSN: Online 1696-3547, Print 0214-6282 
organizer size after gastrulation (Ramel and Lekven 2004; Schier 2001). In addition, Nodal, Fibrobrast growth factors (Fgf), Bone morphogenetic proteins (Bmps) and other factors are required for the formation of the dorsal organizer and correct axis patterning (Belo et al., 2009; Hikasa and Sokol 2013; Langdon and Mullins 2011; Maegawa et al., 2006; Schier 2001; Schier and Talbot 2001; Schier and Talbot 2005).

Bmps, which are members of the TGF- $\beta$ superfamily, exhibit a broad spectrum of biological activities and function as morphogens during embryonic and organ development, contributing to the formation of bone, blood vessels, heart, kidney, neurons, and liver (Langdon and Mullins 2011; Miyazono et al., 2010; Yamamoto and Oelgeschlager 2004). In Xenopus and zebrafish gastrulas, Bmps are distributed in a gradient along the dorsal-ventral axis, where they play an important role in germ layer induction and dorsoventral patterning. In the ectoderm, high levels of Bmp activity specify epidermal fates, intermediate levels lead to the formation of the central nervous system (CNS), and low levels are required for induction of the neural crest. In the mesoderm, high levels of Bmp facilitate formation of the lateral plate mesoderm (LPM), blood tissues, and kidneys, whereas lower Bmp activity is essential for notochord formation (De Robertis and Kuroda 2004; Heasman 2006; Langdon and Mullins 2011; Plouhinec et al., 2013).

Bmp activity gradients are maintained by a large network of molecular regulators that act at the transcriptional and translational levels. The Bmp antagonist Chordin (Chd) contributes to low Bmp activity on the dorsal side of early Xenopus and zebrafish embryos, where it is secreted and binds to Bmp protein, preventing its intracellular signal transduction. In contrast, in Drosophila, the Chordin homolog Sog, is produced on the ventral side of the embryo, where it binds and inhibits diffusion of the Bmp homolog Dpp from the dorsal side of the embryo. The complex is then transported back to the dorsal side of the embryo for degradation (Ben-Zvi et al., 2011; Ben-Zvi et al., 2008; O'Connor et al., 2006; Plouhinec et al., 2011). Other known regulators, such as the metalloproteinase Tolloid, can bind and digest Chd. In addition, twisted Gastrulation
(Tsg) functions extracellularly to facilitate formation of a Chd-TsgBmp trimolecular complex. When this complex flows to the ventral side of the embryo, the high levels of Crossveinless-2 (CV2) there contribute to degradation of Bmp (Plouhinec et al., 2011; Umulis et al., 2009; Zakin and De Robertis 2010).

In early zebrafish gastrulas, different from other bmp genes with the expression in ventral mesoderm, $b m p 2 b$ is also enriched at organizer region exclusively. Its asymmetric expression pattern is initiated by transcriptional repression by bozozok (boz) during the late blastula stage at approximately $4 \mathrm{hpf}$, the expression of boz in the organizer ceases from $50 \%$ epiboly stage onward (Leung et al., 2003; Solnica-Krezel and Driever 2001; Yamanaka et al., 1998). We recently showed that organizer-derived Bmp2b is required for maintenance of the Bmp activity gradient during embryonic development (Xue et al., 2014). However, the elements that mediate transcriptional regulation of organizer-specific $b m p 2 b$ remain unknown.

In this study, we aimed to determine the functional elements that regulate organizer-specific $b m p 2 b$ expression. We generated the novel transgenic line Tsg(-2.272bmp2b:gfp), in which the 2.272-kb $b m p 2 b$ promoter drives organizer-specific $b m p 2 b$ expression. To determine the role of $b m p 2 b$ promoter elements on its transcriptional regulation, we isolated a $0.273-\mathrm{kb}$ minimal promoter from the main 2.272-kb sequence. The minimal promoter, which harbored one consensus Smad-binding element (SBE) and one Lef/Tcf binding element (LBE), responded to Nodal/Smad2 and Wnt/ $\beta$-catenin signal through direct binding of these elements by Smad2 and $\beta$ catenin protein, respectively. Our results indicate that maintenance of organizer-specific bmp2b involves its direct repression by Nodal signal and activation by Wnt signal.

\section{Results}

Zygotic GFP expression of Tsg(-2.272bmp2b:gfp) transgenic
embryos is specifically restricted to dorsal organizer region
We recently reported that a 2,620 -base pair (bp) bmp2b pro-
A
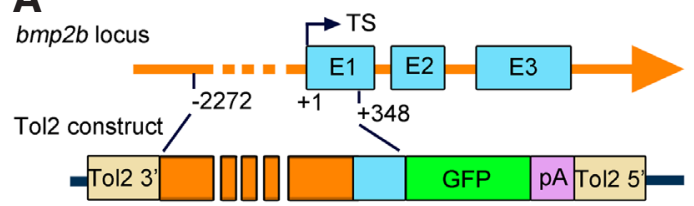

B

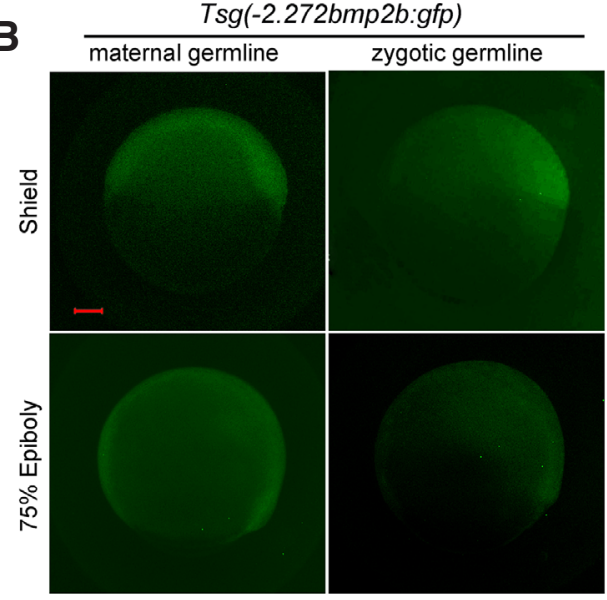

C

Tsg(-2.272bmp2b:gfp) zygotic germline

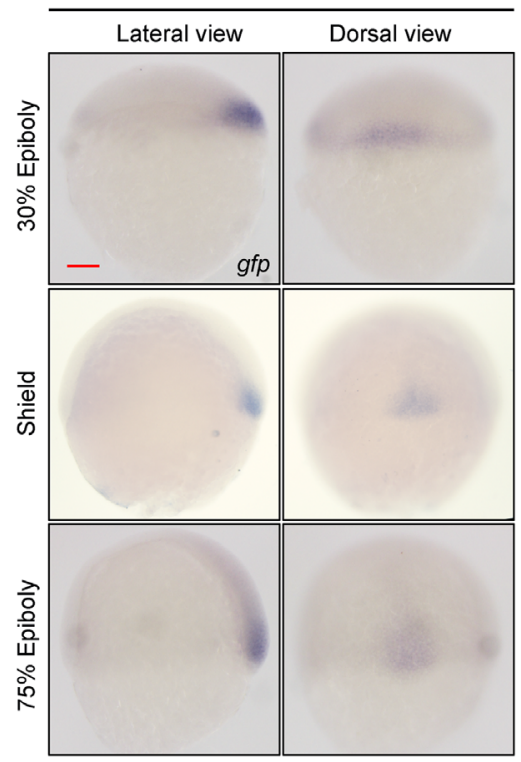

Fig.1. Generation of the organizer-specific bmp2b transgenic line Tsg(-2.272bmp2b:gfp). (A) Schematic diagram of the bmp2b locus and Tol2 construct. The cloned bmp2b promoter $(-2272 \sim+348)$ was inserted into the Tol2 construct for delivery into zebrafish genomic DNA. TS, transcription start site. (B) The GFP expression pattern of the Tsg(-2.272bmp2b:gfp) transgenic line. Embryos derived from Tsg(-2.272bmp2b:gfp) zebrafish crossed with wild-types at shield and $75 \%$ epiboly stage were photographed under fluorescence microscopy. In the maternal germline, embryos were derived from a female transgenic fish crossed with a wild-type male. In the zygotic germline, embryos were generated from a wildtype female crossed with a male transgenic fish. Embryos are displayed laterally, with ventral to the left. (C) Detection of the gfp transcripts by wholemount in situ hybridization. Tsg(-2.272bmp2b:gfp) zygotic germlines were harvested at $30 \%$ epiboly, shield, and $75 \%$ epiboly stage. In the lateral view, the ventral side is oriented to the left. In the dorsal view, the animal pole is oriented to the top. Scale bars, $100 \mu \mathrm{m}$. 
A bmp2b reporter constructs RLA in HEK 293

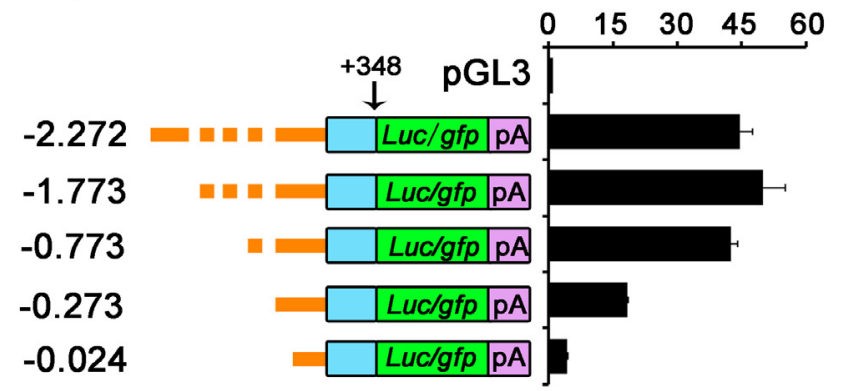

B

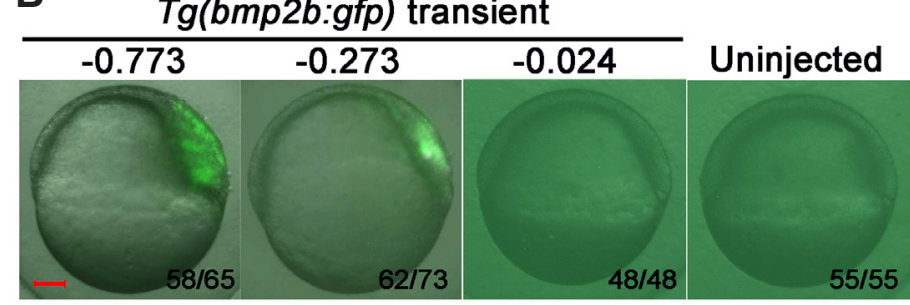

Fig. 2. Deletion analysis of $\boldsymbol{b} \boldsymbol{m} \boldsymbol{m} \mathbf{2} \boldsymbol{b}$ promoter. (A) Illustration of reporter constructs and relative luciferase activity (RLA). Different truncated forms of the bmp $2 \mathrm{~b}$ promoter are shown with the retained promoter length indicated. The luciferase reporters driven by the bmp2b promoter were transfected into HEK293 cells and RLA was detected. (B) Transient GFP expression of indicated reporter constructs in embryos. Constructs containing the truncated form of the bmp2b promoter were injected into the embryo at the one-cell stage and GFP intensity was observed at the shield stage. Views of the embryos are lateral, with dorsal to the right. The ratio of embryos with different GFP expression pattern is indicated. Scale bar, $100 \mu \mathrm{m}$.

moter region, which contains a 2,272-bp sequence upstream of its transcription start site and a 348-bp downstream sequence, has transcriptional activity specific to the dorsal region of the embryo (Xue et al., 2014). To further explore the role of this promoter region on bmp2b expression during development, we isolated this promoter region and inserted it into a Tol2-mediated TSG vector to create a Tsg(-2.272bmp2b:gfp) construct (Fig. 1A). We then used this construct to create a $T s g(-2.272 b m p 2 b: g f p)$ transgenic fish and got five $F_{0}$ founder families, three males and two females, which could stably spread the transgene to next generation. Transgenic screening results showed that the Tsg(-2.272bmp2b:gfp) transgene was maternally expressed, as offspring of heterozygous females bred with wild-type males exhibited ubiquitous GFP expression. In contrast, offspring of heterozygous males bred with wild-type females showed specific but weak GFP expression on the dorsal side at both shield and $75 \%$ epiboly stages (Fig. 1B). We performed in situ hybridization to further characterize the zygotic gfp expression pattern. Dorsal gfp expression was initiated at $30 \%$ epiboly stage, was specifically expressed in the organizer region at shield stage, and was highest at $75 \%$ epiboly stage (Fig. 1C). This expression pattern was consistent with the expression pattern of GFP driven by the same promoter in our recent study (Xue et al., 2014). These results suggest that the 2.272-kb bmp2b promoter region contains regulatory elements required for expression in the organizer region, but lacks elements necessary for expression in other domains, such as the ventral ectoderm. Importantly, the zygotic Tsg(-2.272bmp2b:gfp) germline stably expressed GFP in the dorsal organizer.

\section{Molecular dissection identifies a functional sequence of $\mathrm{bmp} 2 \mathrm{~b}$ promoter for dorsal organizer expression}

In order to determine which region of the 2.272-kb bmp2b promoter is required for organizer-specific $b m p 2 b$ localization, we created a pGL3(-2.272bmp2b:Iuc) construct in which the 2.272$\mathrm{kb}$ bmp2b promoter drives expression of luciferase. A series of truncated forms of the constructs $T g(-2.272 b m p 2 b: g f p)$ and $p G L 3(-$ 2.272bmp2b:luc) were generated to dissect the 2.272-kb bmp2b promoter (Fig. 2). Deletion analysis identified a 0.273-kb proximal promoter region essential for GFP expression in embryos. Injection of the $T g(-0.273 b m p 2 b: g f p)$ plasmid into embryos at the one-cell stage gave rise to transient GFP expression specifically (usually $>80 \%$ ) in the organizer region at the shield stage (Fig. 2B). This truncated promoter also drove transcription of the luciferase reporter in HEK293 cells transfected with the pGL3(-0.273bmp2b:/uc) plasmid (Fig. 2A). Taken together, these data suggest that the $0.273-\mathrm{kb}$ minimal promoter is sufficient to mediate expression of the reporter gene in the organizer region.

\section{Nodal and Wnt signals contribute differently to regulation of organizer-specific bmp2b}

A question of interest to us is how organizer-specific $b m p 2 b$ expression is controlled. We used the pGL3(-0.273bmp2b:luc) construct to perform a luciferase reporter assay in different cell lines. Luciferase expression was repressed by co-transfection of zebrafish Smad3b (Jia et al., 2008) in Hep3B cells, suggesting that Nodal signaling inhibits bmp2b expression. While in HEK293 cells, luciferase expression was enhanced by co-transfection of human Lef1 which recruits $\beta$-catenin to Wnt target genes (Roel et al., 2009) (Fig. 3A), suggesting that canonical Wnt signaling promotes bmp2b expression.

To substantiate the regulatory roles of these two signaling pathways on bmp2bexpression, we knocked down a Nodal co-receptor gene oep, or overexpressed lef1 in wild-type embryos (Gritsman et al., 1999). We then performed in situ hybridization to examine the effects of these manipulations on $b m p 2 b$ transcripts at the shield stage. Knockdown of oep led to an increase in bmp2b expression not only in the organizer but also in the ventral domain (Fig. 3B). Approximately $65 \%$ of embryos in which lef1 was overexpressed showed a similarly increased pattern of bmp2bexpression. The gfp expression in the organizer of Tsg(-2.272bmp2b:gfp)transgenic embryos was also enhanced by oep knockdown or lef1 overexpression (Fig. 3C), consistent with the results from wild-type embryos and the cell reporter assays. These data indicate that organizer-specific bmp2b is inhibited by Nodal signal and activated by canonical Wnt signal. This suggests that the $0.273-\mathrm{kb}$ minimal promoter contains responsive elements for Nodal and Wnt signals.

\section{Transcription of organizer-specific bmp2b is directly down- regulated by Nodal and up-regulated by Wnt signal}

We next asked whether Nodal and Wnt signaling regulate organizer-specific bmp2b transcription directly. To address this question, we searched for Nodal/Smad2/3-responsive and Wnt/3catenin-responsive elements in the $0.273-\mathrm{kb}$ minimal promoter. The $0.273-\mathrm{kb}$ proximal promoter region harbors one putative consensus Smad-binding element (SBE) (CAGAC, -36 -32 bp) (Feng and Derynck 2005) and one putative Lef/Tcf-binding element (LBE) (AGATAA, -263 -258 bp) (Blauwkamp et al., 2008). When the SBE or LBE in the $0.273-k b$ proximal promoter region 
Fig. 3. The 0.273-kb bmp2b dorsal promoter is responsive to Nodal and Wnt/ $\beta$-catenin signaling. (A) Luciferase reporter expression driven by the 0.273$k b$ bmp2b promoter in response to Smad3b or Lef1 stimulation in Hep3B or HEK293 cells. RLA, Relative Luciferase Activity. ${ }^{*} P<0.05 ;{ }^{*} P<0.01 ; N S, P>0.05$. (B,C) Expression of endogenous bmp2b or of gfp from germlineTsg(-2.272bmp2b:gfp) in oep morphants orlef1 overexpressing embryos at the shield stage. Embryos are laterally viewed with dorsal to the right. The ratio of embryos with the representative expression pattern is indicated. std-MO, standard control morpholino; Aslef1 RNA, antisense lef1 mRNA. MO and mRNA were injected at doses: $4 \mathrm{ng}$ for oep-MO and std-MO, $100 \mathrm{pg}$ for lef1 mRNA and Aslef1 mRNA. Scale bar, $100 \mu \mathrm{m}$.

was mutated, luciferase reporter expression in embryos failed to respond to overexpression of constitutively active smad2 (casmad2) (Liu et al., 2013) or to lef1 overexpression (Fig. 4A). Thus, the SBE and LBE are required for the response of the $b m p 2 b$ promoter to Nodal repression and Wnt activation, respectively.

Finally, we used chromatin-immunoprecipitation (ChIP) to investigate whether endogenous Smad2 or $\beta$-catenin could bind the SBE and LBE in the $0.273-\mathrm{kb}$ proximal promoter region in zebrafish embryos. ChIP assays revealed that DNA immunoprecipitated using an anti-Smad2 antibody or an anti- $\beta$-catenin antibody could be amplified using specific primers spanning those elements (Fig. 4B). This indicates that in embryos, the SBE and the LBE regions are bound by Smad 2 and $\beta$-catenin, respectively.

Taken together, our data indicate that expression of $b m p 2 b$ in the organizer is regulated negatively by Nodal/Smad2/3 signaling and positively by $\mathrm{Wnt} / \beta$-catenin signaling in zebrafish embryos.

\section{Discussion}

In this study, we identified a 2.272-kb bmp2bpromoter that drives stable and specific GFP expression in the dorsal organizer region in transgenic gastrulas. In addition, we used molecular dissection
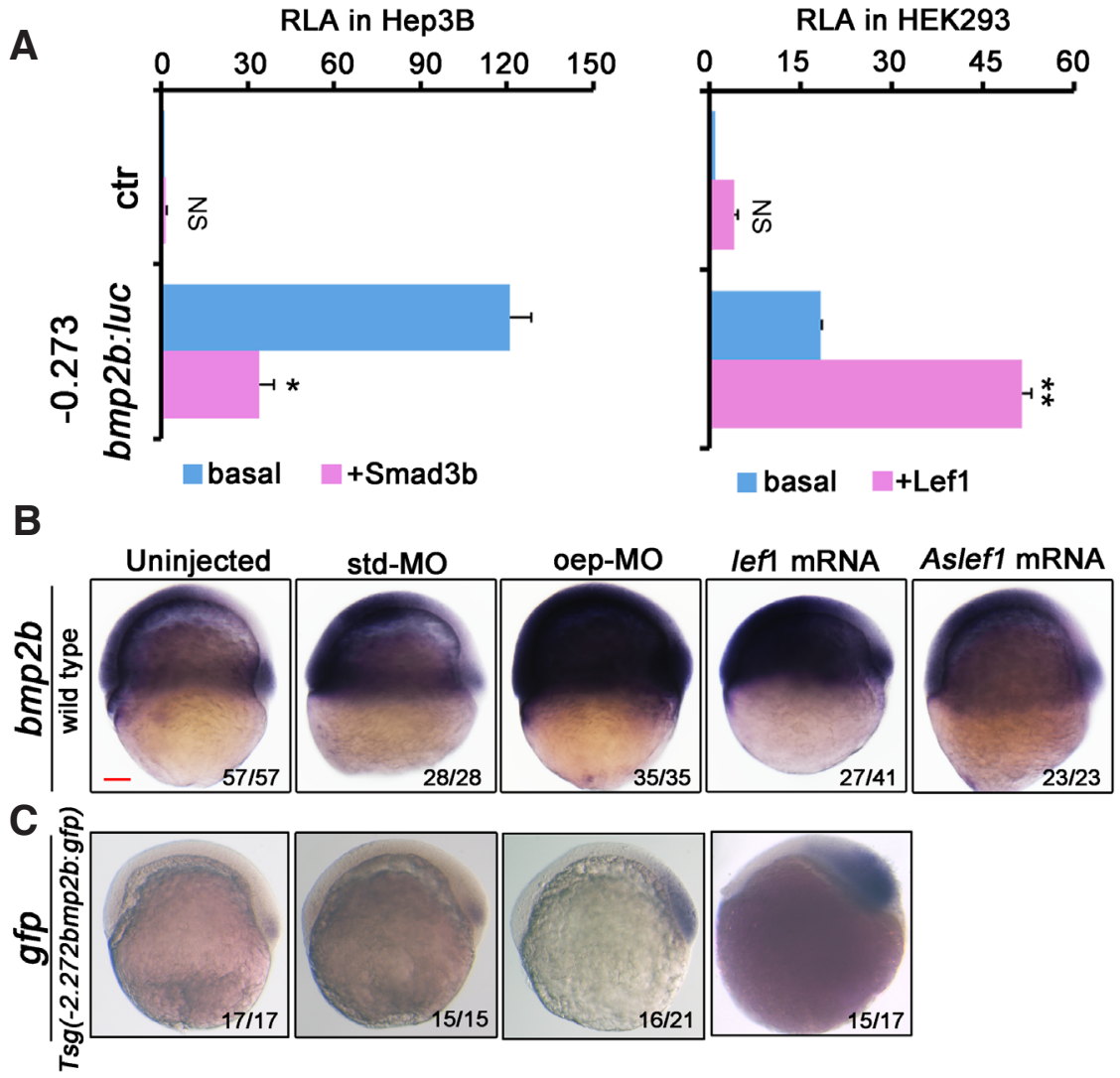

to show that a $0.273-\mathrm{kb}$ minimal promoter was sufficient to express $b m p 2 b$ in the organizer at the shield stage. This $0.273-\mathrm{kb}$ minimal promoter contained a Smad2 binding site and a Lef1 binding site and was directly repressed by Nodal signal and activated by Wnt signal (Fig. 5).

In zebrafish, bmp2b mRNA is observed in the organizer region beginning at around $5.7 \mathrm{hpf}$, at the onset of organizer formation. bmp2b mRNA is further enriched in the organizer region and the dorsal side of the embryo throughout the gastrula stage. As mentioned earlier in the text, boz, a gene downstream of the Wnt signaling pathway, acts as the earliest repressor of $b m p 2 b$ tran-

A

$-0.273 b m p 2 b: L u c$
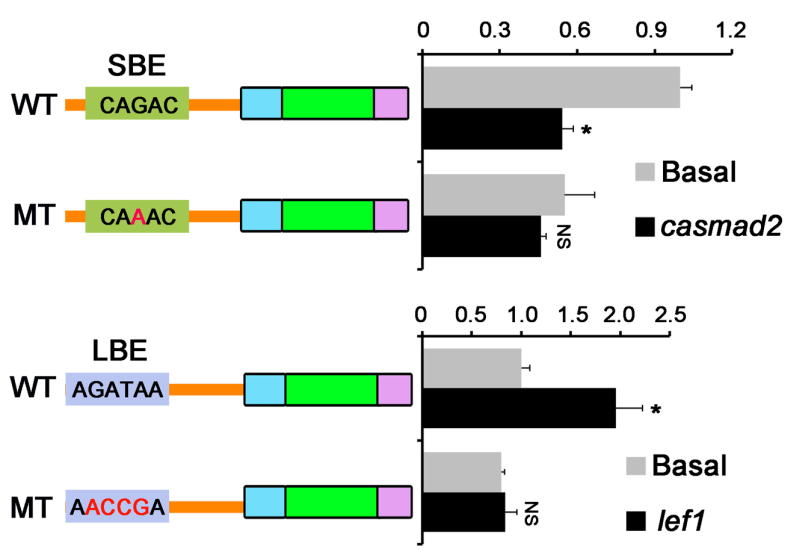

B

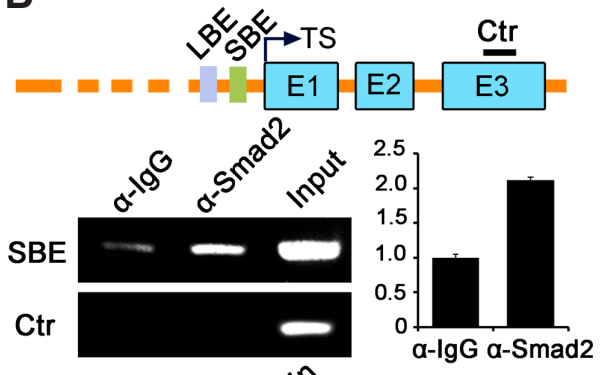

LBE

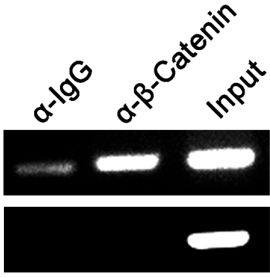

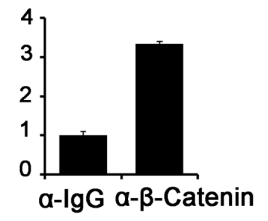

Fig. 4. The 0.273-kb bmp2b dorsal promoter is directly controlled by Nodal and Wnt/ $\beta$-catenin signaling. (A) Effect of mutations in SBE or LBE of the 0.273-kb bmp2b promoter on luciferase reporter expression. The construct structures are illustrated on the left and RLA in injected embryos at the shield stage are shown on the right. (B) Smad2 or $\beta$-catenin occupancy of the bmp $2 \mathrm{~b}$ promoter. On the top, illustration of the amplified regions after ChIP; bottom, gel images (left) and quantification (right) of ChIP-PCR products. * $P<0.05$; ${ }^{*} P<0.01 ; N S, P>0.05$. 

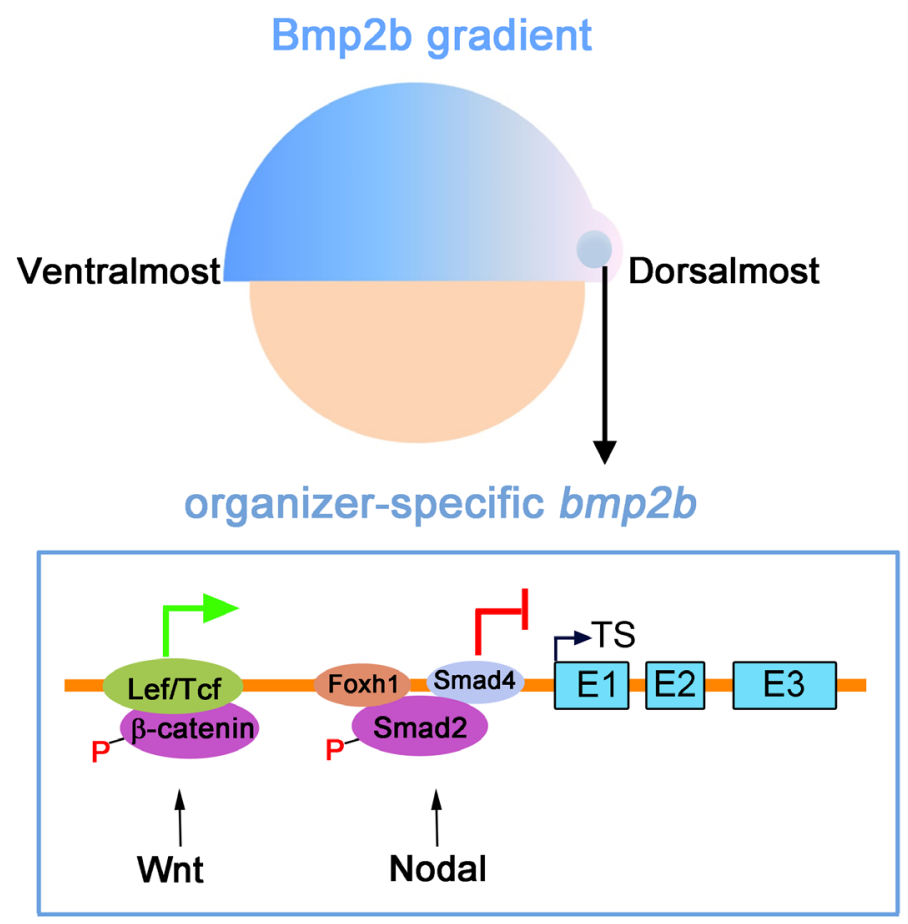

Fig. 5. Molecular regulatory model of organizer-specific bmp2b. Top image shows Bmp2b distribution at the shield stage. Embryos are viewed laterally with dorsal to the right. Bmp2b forms a gradient distribution along the ventral-dorsal axis, with the highest expression ventralmost, a gradual reduction in expression on the dorsal side, and specific enrichment at the dorsal organizer. Bottom image shows transcriptional regulation of organizer-specific bmp2b is mediated through activation by canonical Wnt signaling via Lef/Tcf1 binding, and repression by Nodal signaling via Smad2 binding on its promoter.

scription in the dorsal field by directly binding to the $b m p 2 b$ intron in the late blastula. When the organizer forms, Boz expression ceases, releasing repression of $b m p 2 b$ and promoting its renewed expression in the organizer (Leung et al., 2003; Solnica-Krezel and Driever 2001). Nodal and Wnt signals are required for organizer formation, and their functions are conserved across vertebrates (Schier 2001). Here, we focused on the transcriptional regulation of organizer-specific $b m p 2 b$ at the shield stage. Our results confirmed that Nodal and Wnt signals are involved in the maintenance of organizer-specific bmp2b expression through direct binding of a Smad-binding site and Lef-binding site, respectively, in the 0.273-kb promoter (Fig. 4). The functional effects of Nodal and Wnt signals on organizer-specific $b m p 2 b$ transcriptional regulation were opposite of one another, with Nodal signal repressing $b m p 2 b$ expression and Wnt signal activating it. The interaction between these two signals deserves further investigation.

The 2.272-kb bmp2bpromoter initiated gfpreporter gene expression in the dorsal side of the embryo at $30 \%$ epiboly stage (Fig. $1 C)$. This was earlier than initiation of endogenous dorsal bmp2b expression (Xue et al., 2014), suggesting that the 2.272-kb promoter lacks the binding elements of transcriptional repressor such as Boz, which is necessary for inhibition of dorsal bmp2b transcription at the early gastrula stage through targeting its first intron (Leung et al., 2003). We also deleted a $50 \mathrm{bp}$ region (-123 -74 bp) of the $0.273-\mathrm{kb}$ promoter to further explore its regulation. This deletion led to the permanent absence of gfp expression (data not shown), suggesting that an important enhancer is embedded within this 50 bp sequence. As Fgf signal and other factors are essential for organizer formation (Kuo et al., 2013; Maegawa et al., 2006), it is likely that other signals or transcriptional factors work together with Nodal and Wnt signals to regulate organizer-specific bmp2b transcription. This hypothesis should be investigated in future studies.

One interesting result was that mutation of SBE included in the $0.273-\mathrm{kb} b m p 2 b$ promoter caused almost loss of responsiveness to casmad2 repression. While, this mutation also reduced the luciferase reporter expression in embryos at basal level (Fig. $4 A$ ). This phenomenon may be explained that perhaps SBE not only functions as a repressor, but also an enhancer for some other factors, that is, there may exist some co-activators essential for transcriptional activity, which can form complex with Smad2/3 to function. Mutation of SBE both suppresses the binding of the complex to SBE and down-regulates the transcriptional activity of reporter gene.

As we mentioned in the introduction, maternal and zygotic Wnt signal function differently during dorsoventral patterning, previous study suggested that Wnt signal is inactive at the dorsal margin during late gastrulation (Lu et al., 2011; Ramel and Lekven 2004; Shimizu et al., 2012). One interesting issue is if Wnt signal continuously activates the expression of $b m p 2 b$ at dorsal margin after shield stage? To explore this, we incubated wild-type embryos with a Wnt inhibitor, IWR-1-endo (1:5000, Selleck) (Burridge etal., 2014) from 64-cell stage. Compared with control group treated with DMSO, $100 \%$ of embryos treated with IWR showed observably reduced bmp2b expression in organizer region at shield stage (Fig. S1A), while not clearly decreased at dorsal margin at $75 \%$ epiboly stage (Fig. S1B). These results suggested that Wnt-signal is essential for $b m p 2 b$ expression in organizer region but becomes weak and contributes less to bmp2b expression at dorsal margin during late gastrulation. We speculate that other genes expressing in this region compensate for the dorsal $b m p 2 b$ maintenance at these stages.

In conclusion, we showed that organizer-specific bmp2b, which is essential for maintenance of the Bmp2b activity gradient, and more broadly, for dorsoventral patterning, was regulated by Nodal and Wnt signals in an opposite manner, thereby maintaining a balance of $b m p 2 b$ expression in the dorsal organizer.

\section{Materials and Methods}

\section{Zebrafish embryos and cell lines}

Zebrafish embryos, the human 293 cell line, and the Hep3B cell line were used in this study. All animal studies were performed in accordance with guidelines approved by the institutional Human Ethics Review Committee and the Animal Care and Use Committee of Tsinghua University.

\section{Molecular cloning}

A sequence between base pairs -2272 to +348 of the bmp2b locus was amplified by PCR from zebrafish genomic DNA and cloned into the $p G L 3$ basic vector (Promega) to form the construct $p G L 3(-2.272 b m p 2 b: l u c)$. This insert was then subcloned into a modified version of the $p G L 3$ basic vector in which Luciferase was replaced by the enhanced green fluorescent protein (GFP) coding sequence to generate the construct $T g(-2.272 b m p 2 b: g f p)$ for transient expression and deletion analysis. The primer sequences used for PCR amplification of DNA were the same as those used previously (Xue et al., 2014).

To create the construct for antisense lef1 mRNA, lef1 coding sequence was amplified by PCR from cDNA, then was reversely inserted into $p X T 7$ 
vector. The primer sequences were: Forward-Spel: 5'-GGACTAGTatgccgcagttgtcaggtgga-3'; Reverse-EcoRI: 5'-CCGGAATTCtcagatgtacgccgttttcattc-3'.

\section{Zebrafish strains and transgenesis}

The tuebingen zebrafish strain was used in this study. To generate the transgenic line, the $b m p 2 b$ promoter was cloned into a modified TSG vector (a map of the modified TSG vector used in this study can be provided upon request) (Han et al., 2011). The plasmid Tsg(-2.272bmp2b:gfp) was then co-injected with Tol2 transposase mRNA into one-cell wild-type embryos. These founder fish were then mated to wild-type fish, and progeny that carried the transgene were identified by GFP expression. Embryos born from male founders showed specific but weak GFP expression that could be detected by in situ hybridization, while offspring of female founders displayed strong maternal GFP expression.

\section{RNA synthesis, morpholinos, microinjection and whole-mount in situ hybridization}

mRNAs were synthesized in vitrofrom corresponding linearized plasmids using the mMessage mMachine kit (Ambion). Digoxigenin-UTP-labeled antisense RNA probes were generated by in vitro synthesis with a linearized plasmid as a template (Roche).

Whole-mount in situ hybridization was performed as previously described (Xiong et al., 2006). Stained embryos were cleared in glycerol and photographed using a digital camera (SPOT Insight) under a Nikon SMZ 1500 microscope. The images were adjusted using Adobe Photoshop software.

The morpholinos used in this study were synthesized by Gene Tools, LLC and was as follows: oep-MO, 5'-GCCAATAAACTCCAAAACAACTCGA-3' (Nasevicius and Ekker 2000). standard-MO, 5'-AAGGAAAAAACGAAATGGAAAGGAT-3'.

Approximately 1-1.5 $\mathrm{nl}$ of morpholino solution or DNA was injected into the yolk or cell of each embryo at the one-cell stage using MPPI-2 quantitative injection equipment (Applied Scientific Instrumentation Co.).

\section{Luciferase reporter and chromatin immunoprecipitation assay}

Cell culture, transfection, and luciferase reporter assays were performed as previously described (Liu et al., 2013), Shield stage embryos were harvested for embryonic reporter assays.

The chromatin-immunoprecipitation (ChIP) assay was performed as described previously (Liu et al., 2011). For this experiment, approximately 1500 embryos at shield stage were harvested. Arabbit anti-Smad2/3 antibody (Cell Signaling, 1:50) and a rabbit anti- $\beta$-catenin antibody (Abcam, 1:200) were used. The primer sequences for ChIP-PCR were: bmp2b-forward: 5'-ATACAAATGTAGATAATTTA-3'; bmp2b-reverse: 5'-TTTTTGTTGTCTGATGATGT-3'; control-forward: 5'-ACACCCGTCTGGTGCAGGAC-3'; control-reverse:5'-AGCCTCCTCGGATACTTC-3'. The ChIP-PCR products were examined by gel electrophoresis and band intensity was quantified using ImageJ software.

\section{Statistical analysis}

Student's $t$-tests (two-tailed, unequal variance) were used to determine p-values of all groups compared in this study. Significance levels were donated as ${ }^{*} p<0.05$ and ${ }^{* *} p<0.01$

\section{Acknowledgement}

We are grateful to the members of the Meng Laboratory and Pan Laboratory for discussion and technical assistance, and to Dr. Alexander Schier for helpful discussion and suggestions. This work was financially supported by grants from the National Natural Science Foundation of China (\#31501174) and from Natural Scinece Foundation of Fujian Province (\#2015J05070) and from Outstanding Youth Research Personnel Training Program of Minnan Normal University (MJ14007).

\section{Author Contributions}

Conceived and designed the experiments: YX CX AM YP. Performed the experiments: $Y X C X C C W Z J X$. Analyzed the data: $Y X C X$ AM. Contributed reagents/materials/analysis tools: YX CX AM YP. Wrote the paper: YX CX AM.

\section{References}

BELO, J. A., SILVA, A. C., BORGES, A. C., FILIPE, M., BENTO, M., GONCALVES, L., VITORINO, M., SALGUEIRO, A. M., TEXEIRA, V., TAVARES, A. T. and MARQUES, S. (2009). Generating asymmetries in the early vertebrate embryo: the role of the Cerberus-like family. Int J Dev Biol 53: 1399-1407.

BEN-ZVI, D., SHILO, B. Z. and BARKAI, N. (2011). Scaling of morphogen gradients. Curr Opin Genet Dev 21: 704-710.

BEN-ZVI, D., SHILO, B. Z., FAINSOD, A. and BARKAI, N. (2008). Scaling of the BMP activation gradient in Xenopus embryos. Nature 453: 1205-1211.

BLAUWKAMP, T. A., CHANG, M. V. and CADIGAN, K. M. (2008). Novel TCF-binding sites specify transcriptional repression by Wnt signalling. EMBO J27: 1436-1446.

BURRIDGE, P. W., METZLER, S. A., NAKAYAMA, K. H., ABILEZ, O. J., SIMMONS, C. S., BRUCE, M. A., MATSUURA, Y., KIM, P., WU, J. C., BUTTE, M., HUANG, N. F. and YANG, P. C. (2014). Multi-cellular interactions sustain long-term contractility of human pluripotent stem cell-derived cardiomyocytes. Am J Trans/ Res 6: 724-735.

DE ROBERTIS, E. M. and KURODA, H. (2004). Dorsal-ventral patterning and neural induction in Xenopus embryos.Ann. Rev. Cell Dev. Biol. 20: 285.

FENG, X. H. and DERYNCK, R. (2005). Specificity and versatility in tgf-beta signaling through Smads. Annu Rev Cell Dev Biol 21: 659-693.

GRITSMAN, K., ZHANG, J., CHENG, S., HECKSCHER, E., TALBOT, W. S. and SCHIER, A. F. (1999). The EGF-CFC protein one-eyed pinhead is essential for nodal signaling. Cell 97: 121-132.

HAN, Y., MU, Y., LI, X., XU, P., TONG, J., LIU, Z., MA, T., ZENG, G., YANG, S., DU, J. and MENG, A. (2011). Grhl2 deficiency impairs otic development and hearing ability in a zebrafish model of the progressive dominant hearing loss DFNA28. Hum Mol Genet 20: 3213-3226.

HARLAND, R. and GERHART, J. (1997). Formation and function of Spemann's organizer. Annu Rev Cell Dev Biol 13: 611-667.

HEASMAN, J. (2006). Patterning the early Xenopus embryo. Development 133 1205-1217.

HIKASA, H. and SOKOL, S. Y. (2013). Wnt signaling in vertebrate axis specification. Cold Spring Harb Perspect Biol 5: a007955.

JIA, S., REN, Z., LI, X., ZHENG, Y. and MENG, A. (2008). smad2 and smad3 are required for mesendoderm induction by transforming growth factor-beta/nodal signals in zebrafish. J. Biol. Chem. 283: 2418-2426.

KELLY, C., CHIN, A. J., LEATHERMAN, J. L., KOZLOWSKI, D. J. and WEINBERG, E. S. (2000). Maternally controlled (beta)-catenin-mediated signaling is required for organizer formation in the zebrafish. Development 127: 3899-3911.

KUO, C. L., LAM, C. M., HEWITT, J. E. and SCOTTING, P. J. (2013). Formation of the embryonic organizer is restricted by the competitive influences of $\mathrm{Fg}$ signaling and the SoxB1 transcription factors. PLoS One 8: e57698.

LANGDON, Y. G. and MULLINS, M. C. (2011). Maternal and zygotic control of zebrafish dorsoventral axial patterning. Annu Rev Genet 45: 357-377.

LEUNG, T., BISCHOF, J., SÖLL, I., NIESSING, D., ZHANG, D., MA, J., JÄCKLE, H. and DRIEVER, W. (2003). bozozok directly represses bmp2b transcription and mediates the earliest dorsoventral asymmetry of bmp2b expression in zebrafish. Development 130: 3639-3649.

LIU, X., XIONG, C., JIA, S., ZHANG, Y., CHEN, Y. G., WANG, Q. and MENG, A. (2013). Araf kinase antagonizes Nodal-Smad2 activity in mesendoderm development by directly phosphorylating the Smad2 linker region. Nat Commun 4: 1728 .

LIU, Z., LIN, X., CAI, Z., ZHANG, Z., HAN, C., JIA, S., MENG, A. and WANG, Q. (2011). Global identification of SMAD2 target genes reveals a role for multiple coregulatory factors in zebrafish early gastrulas. J. Biol. Chem. 286: 28520-28532.

LU, F. I., THISSE, C. and THISSE, B. (2011). Identification and mechanism of regulation of the zebrafish dorsal determinant. Proc Natl Acad Sci USA 108: 15876-15880.

MAEGAWA, S., VARGA, M. and WEINBERG, E. S. (2006). FGF signaling is required for \{beta\}-catenin-mediated induction of the zebrafish organizer. Development 133: 3265-3276

MEDINA, A., WENDLER, S. and STEINBEISSER, H. (1997). Cortical rotation is required for the correct spatial expression of $\mathrm{nr} 3$, sia and gsc in Xenopus embryos. 
Int J Dev Biol 41: 741-745.

MIYAZONO, K., KAMIYA, Y. and MORIKAWA, M. (2010). Bone morphogenetic protein receptors and signal transduction.J Biochem 147: 35-51.

NASEVICIUS, A. and EKKER, S. C. (2000). Effective targeted gene 'knockdown' in zebrafish. Nat Genet 26: 216-220.

O'CONNOR, M. B., UMULIS, D., OTHMER, H. G. and BLAIR, S. S. (2006). Shaping BMP morphogen gradients in the Drosophila embryo and pupal wing. Development 133: 183-193.

PLOUHINEC, J. L., ZAKIN, L. and DE ROBERTIS, E. M. (2011). Systems control of BMP morphogen flow in vertebrate embryos. Curr Opin Genet Dev 21: 696-703.

PLOUHINEC, J. L., ZAKIN, L., MORIYAMA, Y. and DE ROBERTIS, E. M. (2013). Chordin forms a self-organizing morphogen gradient in the extracellular space between ectoderm and mesoderm in the Xenopus embryo. Proc Natl Acad Sci USA 110: 20372-20379.

RAMEL, M. C. and LEKVEN, A. C. (2004). Repression of the vertebrate organizer by Wnt8 is mediated by Vent and Vox. Development 131: 3991-4000.

ROEL, G., GENT, Y. Y., PETERSON-MADURO, J., VERBEEK, F. J. and DESTREE, O. (2009). Lef1 plays a role in patterning the mesoderm and ectoderm in Xenopus tropicalis. Int J Dev Biol 53: 81-89.

SCHIER, A. F. (2001). Axis formation and patterning in zebrafish. Curr Opin Genet Dev 11: 393-404.

SCHIER, A. F. and TALBOT, W. S. (2001). Nodal signaling and the zebrafish organizer. Int J Dev Biol 45: 289-297.

SCHIER, A. F. and TALBOT, W. S. (2005). Molecular genetics of axis formation in zebrafish. Annu Rev Genet 39: 561-613.

SHIMIZU, N., KAWAKAMI, K. and ISHITANI, T. (2012). Visualization and exploration of Tcf/Lef function using a highly responsive Wnt/beta-catenin signaling-reporter transgenic zebrafish. Dev Biol 370: 71-85.

SOLNICA-KREZEL, L. and DRIEVER, W. (2001). The role of the homeodomain protein Bozozok in zebrafish axis formation. Int J Dev Biol 45: 299-310.

UMULIS, D., O'CONNOR, M. B. and BLAIR, S. S. (2009). The extracellular regulation of bone morphogenetic protein signaling. Development 136: 3715-3728.

XIONG, B., RUI, Y., ZHANG, M., SHI, K., JIA, S., TIAN, T., YIN, K., HUANG, H., LIN, S., ZHAO, X., CHEN, Y., CHEN, Y. G., LIN, S. C. and MENG, A. (2006). Tob1 controls dorsal development of zebrafish embryos by antagonizing maternal beta-catenin transcriptional activity. Dev Cell 11: 225-238.

XUE, Y., ZHENG, X., HUANG, L., XU, P., MA, Y., MIN, Z., TAO, Q., TAO, Y. and MENG, A. (2014). Organizer-derived Bmp2 is required for the formation of a correct Bmp activity gradient during embryonic development. Nat Commun 5: 3766 .

YAMAMOTO, Y. and OELGESCHLAGER, M. (2004). Regulation of bone morphogenetic proteins in early embryonic development. Naturwissenschaften 91:519-534.

YAMANAKA, Y., MIZUNO, T., SASAI, Y., KISHI, M., TAKEDA, H., KIM, C. H., HIBI, M. and HIRANO, T. (1998). A novel homeobox gene, dharma, can induce the organizer in a non-cell-autonomous manner.Genes Dev 12: 2345-2353.

YASUO, H. and LEMAIRE, P. (2001). Generation of the germ layers along the animalvegetal axis in Xenopus laevis. Int J Dev Biol 45: 229-235.

ZAKIN, L. and DE ROBERTIS, E. M. (2010). Extracellular regulation of BMP signaling. Curr Biol 20: R89-92. 


\section{Further Related Reading, published previously in the Int. J. Dev. Biol.}

The Lophotrochozoan TGF- $\beta$ signalling cassette - diversification and conservation in a key signalling pathway Nathan J. Kenny,Erica K.O. Namigai, Peter K. Dearden, Jerome H.L. Hui, Cristina Grande and Sebastian M. Shimeld Int. J. Dev. Biol. (2014) 58: 533-549

http://www.intjdevbiol.com/web/paper/140080nk

KDEL tagging: a method for generating dominant-negative inhibitors of the secretion of TGF-beta superfamily proteins Shinya Matsukawa, Yuki Moriyama, Tadayoshi Hayata, Haruka Sasaki, Yuzuru Ito, Makoto Asashima and Hiroki Kuroda Int. J. Dev. Biol. (2012) 56: 351-356

http://www.intjdevbiol.com/web/paper/123514sm

Visualization, characterization and modulation of calcium signaling during the development of slow muscle cells in intact zebrafish embryos

Chris Y. Cheung, Sarah E. Webb, Donald R. Love and Andrew L. Miller

Int. J. Dev. Biol. (2011) 55: 153-174

http://www.intjdevbiol.com/web/paper/103160cc

Hematopoietic development in the zebrafish

Elizabeth J. Paik and Leonard I. Zon

Int. J. Dev. Biol. (2010) 54: 1127-1137

http://www.intjdevbiol.com/web/paper/093042ep

The mob as tumor suppressor (mats1) gene is required for growth control in developing zebrafish embryos

Yuan Yuan, Shuo Lin, Zuoyan Zhu, Wenxia Zhang and Zhi-Chun Lai

Int. J. Dev. Biol. (2009) 53: 525-533

http://www.intjdevbiol.com/web/paper/082720yy

Expression dynamics of the LIM-homeobox genes, Lhx1 and Lhx9, in the diencephalon during chick development

Xiangnan Sun, Hirotomo Saitsu, Kohei Shiota and Makoto Ishibashi

Int. J. Dev. Biol. (2008) 52: 33-41

http://www.intjdevbiol.com/web/paper/072386xs

Frontiers in fluorescence microscopy

José Rino, José Braga, Ricardo Henriques and Maria Carmo-Fonseca

Int. J. Dev. Biol. (2009) 53: 1569-1579

http://www.intjdevbiol.com/web/paper/072351jr

5 yr ISI Impact Factor $(2013)=2.879$
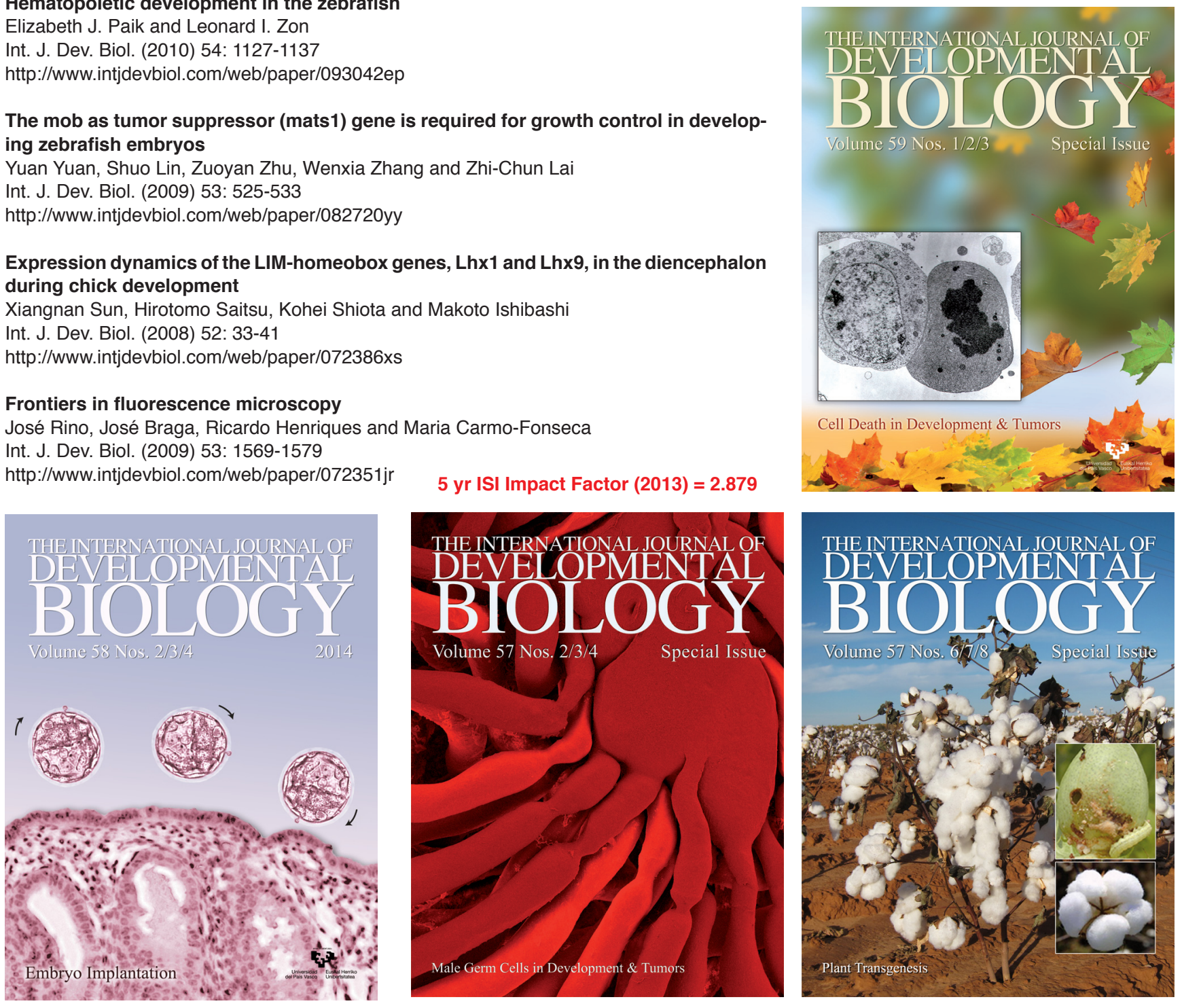